\title{
Comunicação
}

[Communication]

\section{Influência do parasitismo por nematódeos sobre o perfil hematológico de caprinos}

[Influence of nematodes on the hematological parameters of goats]

\author{
M.J.T. Mattos, C.M.B. Oliveira, A. Lustosa, L.A. Lacerda, S. Terra \\ Faculdade de Veterinária - Universidade Federal do Rio Grande do Sul \\ Av. Bento Gonçalves, 9090 \\ 91540.000 - Porto Alegre, RS
}

\begin{abstract}
A principal característica da infecção gastrintestinal por helmintos é a severa depressão da capacidade digestiva e de absorção na mucosa do local da infecção. Estudos sobre o impacto das nematodeoses em caprinos foram relatados na Austrália (Rahman e Collins, 1991), França (Hoste et al., 2001 apud Hoste et al., 2002), África (Vatta et al., 2002), Brasil (Costa et al., 2000). As informações sobre as alterações hematológicas, em caprinos parasitados por nematódeos, são escassas no Brasil. O presente experimento objetivou correlacionar os parâmetros hematológicos entre caprinos naturalmente parasitados por nematódeos com os não parasitados e o efeito da medicação com ivermectina, no Rio Grande do Sul.
\end{abstract}

Foram selecionados 36 caprinos, sem raça definida, previamente avaliados quanto à eficácia do princípio ativo e provenientes de duas propriedades da Grande Porto Alegre, RS. Eles tinham quatro meses de idade, $9,83 \mathrm{~kg}$ de peso médio, sendo: 12 animais naturalmente parasitados por nematódeos gastrintestinais da super família Trichostrongyloidea sensíveis à ivermectina (G1s), 12 parasitados por nematódeos resistentes à ivermectina (G2r) e 12 não parasitados (G3 np). Dos parasitados, seis caprinos de cada grupo foram medicados com ivermectina na dose de $200 \mu \mathrm{g} / \mathrm{kg}$ (G1sm e G2rm), via oral, e seis de cada grupo, não foram medicadas e serviram como controle (G1sc e G2rc). Amostras de fezes e de sangue foram coletadas no dia da medicação (dia zero) e sete e
14 dias após. Nas amostras de fezes foram realizadas contagens de ovos por grama de fezes (OPG) por meio do método de Gordon e Whitlock (1939) e cultura de larvas segundo Roberts e O'Sullivan (1950), citados por Ueno e Gonçalves (1998). Amostras individuais de sangue foram coletadas em tubos tipo vacutainer, de $3 \mathrm{ml}$, com anticoagulante e analisadas conforme as técnicas de microematócrito, colorímetro para dosagem de hemoglobina e contagem de células na câmara de Neubauer. Foram realizadas análise da variância e de correlação parcial utilizando o software SPSS (Montgomery, 1997).

Os resultados segundo os grupos (tratamentos) são apresentados na Tab. 1. No OPG de Trichostrongyloidea a redução atribuída à medicação foi de $93,2 \%$ para o grupo G1sm e de 42,1 para o G2rm

Os valores de eritrócitos e de hematócrito em caprinos parasitados com estirpe sensível ao Haemonchus foram superiores aos observados no grupo parasitado por estirpe resistente $(\mathrm{P}=0,0002$ e $\mathrm{P}=0,006$, respectivamente ). Estes valores são inferiores quando comparados aos observados em caprinos não parasitados. Observou-se correlação inversa entre OPG e hematócrito $(\mathrm{r}=-0,498 ; \mathrm{P}<0,13)$. Vatta et al. (2002), na África, e Costa et al. (2000), no Brasil, também constataram correlação negativa entre essas variáveis. Segundo esses autores, as alterações no hematócrito e na hemoglobina são fatores

Recebido para publicação em 23 de julho de 2003

Recebido para publicação, após modificações, em 6 de maio de 2004

E-mail: mary.gomes@ufrgs.br 
marcantes nos hospedeiros resistentes, servindo como parâmetros para avaliar a resistência em caprinos. O gênero Haemonchus foi o mais prevalente em todos os grupos parasitados, indicando que as alterações hematológicas observadas foram decorrentes, em especial, do parasitismo por esse helminto. O perfil hematológico não foi alterado em função da medicação anti-helmíntica. Nas condições deste experimento, os caprinos que albergaram a estirpe resistente à aplicação de ivermectina sofreram mais a ação do parasitismo.

Tabela 1. OPG, número de larvas de Haemonchus e Ostertagia, não resistentes e resistentes, valores de eritrócitos, hemoglobina e hematrócrito em caprinos parasitados e tratados com ivermectina

\begin{tabular}{|c|c|c|c|}
\hline \multirow{2}{*}{ Variável } & \multicolumn{3}{|c|}{ Dias após a medicação } \\
\hline & 0 & 7 & 14 \\
\hline \multicolumn{4}{|c|}{ OPG de Trichostrongyloidea } \\
\hline G1sm & 3658 & 125 & 250 \\
\hline G1sc & 3000 & 3200 & 3700 \\
\hline G2rm & 3583 & 1133 & 3050 \\
\hline $\mathrm{G} 2 \mathrm{rc}$ & 3100 & 2850 & 5267 \\
\hline \multicolumn{4}{|c|}{ Larvas de Haemonchus (\%) } \\
\hline G1sm & 74 & 100 & 100 \\
\hline G1sc & 71 & 81 & 75 \\
\hline G2rm & 95 & 89 & 93 \\
\hline $\mathrm{G} 2 \mathrm{rc}$ & 95 & 97 & 97 \\
\hline \multicolumn{4}{|c|}{ Larvas de Ostertagia (\%) } \\
\hline G1sm & 26 & 0 & 0 \\
\hline G1sc & 29 & 19 & 25 \\
\hline G2rm & 5 & 11 & 7 \\
\hline $\mathrm{G} 2 \mathrm{rc}$ & 5 & 3 & 3 \\
\hline \multicolumn{4}{|c|}{ Eritrócitos $\left(\times 10^{6} / \mathrm{mm}^{3}\right)$} \\
\hline G1sm & 14,08 & 15,04 & 13,70 \\
\hline G1sc & 13,31 & 14,30 & 13,96 \\
\hline $\mathrm{G} 2 \mathrm{rm}$ & 11,17 & 10,37 & 10,68 \\
\hline G2rc & 12,38 & 11,14 & 9,90 \\
\hline G3np & 15,78 & 15,77 & 15,78 \\
\hline \multicolumn{4}{|c|}{ Hemoglobina (mg/dl) } \\
\hline G1sm & 10,35 & 10,30 & 9,89 \\
\hline G1sc & 10,00 & 9,51 & 9,48 \\
\hline G2rm & 9,20 & 8,94 & 10,72 \\
\hline G2rc & 9,58 & 9,34 & 8,12 \\
\hline G3np & 11,00 & 11,33 & 11,50 \\
\hline \multicolumn{4}{|c|}{ Hematócrito (\%) } \\
\hline G1sm & 30,3 & 33,0 & 29,5 \\
\hline G1sc & 28,7 & 30,8 & 25,8 \\
\hline $\mathrm{G} 2 \mathrm{rm}$ & 25,2 & 22,3 & 23,0 \\
\hline G2rc & 26,7 & 24,0 & 21,3 \\
\hline G3np & 30,8 & 34,0 & 34,0 \\
\hline
\end{tabular}

Palavras-chave: caprinos, Haemonchus, resistência e sensibilidade, hematologia, ivermectina 


\begin{abstract}
The interaction between ivermectin-resistant and ivermectin-sensitive nematodes and the effect of this anthelmintic on the hematological status of naturally infected goats was assessed using 36 animals. Of these animals, 12 were infected by ivermectin-sensitive gastrointestinal nematodes of the superfamily Trichostrongyloidea (G1s e G1s) 12 were infected by ivermectin-resistant nematodes (G2r e G2r) and 12 uninfect goats (G3np). Six infected goats of each group (G1sm e G2rm) received oral ivermectin al the

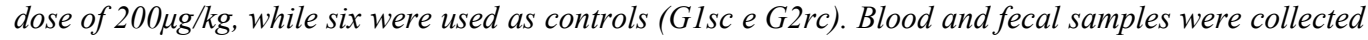
on the day of medication (day zero), at seven, and at 14 days thereafter. The erythrocyte count and hematocrit levels in goats infected by sensitive strains of Haemonchus was greater than that of the group infected by a resistant strain. These values are lower in comparison to those observed in uninfected goats. The increase in the number of eggs per gram of feces, regardless of the strain, is inversely related to the hematocrit level. The use of ivermectin did not significantly change the hematological parameters of goats.
\end{abstract}

Keywords: goat, Haemonchus, sensitive-resistant, hematology, ivermectin

\section{REFERÊNCIAS BIBLIOGRÁFICAS}

COSTA, C.A.F.; VIEIRA, L.D.A.; BERNE, M.E.A. et al. Variability of resistance in goats infected with Haemonchus contortus in Brazil. Vet. Parasitol., v.88, p.153-158, 2000.

HOSTE, H.; LE FRILEUX,Y.; POMMARET, A. Comparison of selective and systematic treatments to control nematode infection of the digestive tract in dairy goats. Vet.Parasitol., v.106, p.257-355, 2002.

MONTGOMERY, D.C. Design and analysis of experiments. New York: John Wiley \& Sons, 1997. 704p.
RAHMAN, W.A.; COLLINS, G.H. Changes in live weight gain, blood constituents and worm egg goats articially infected with sheep-strain of Haemonchus contortus. Br. Vet. J., v.146, p.543$550,1991$.

UENO, H.; GONÇALVES, P.C. Manual para diagnóstico das helmintoses de ruminantes. 4.ed. JICA, 1998. 166p.

VATTA, A.F.; KRECEK, R.C.; LETTY, B.A. et al. Incidence of Haemonchus spp and effect on haematocrit and eye colour in goats farmed under resource-poor conditions in South Africa. Vet. Parasitol., v.103, p.119-131, 2002. 